\title{
Coverings, Laplacians, and Heat Kernels of Directed Graphs
}

\author{
Clara E. Brasseur* \\ Oberlin College \\ 52 West Lorain Street \\ Oberlin $\mathrm{OH} 44074$ \\ Clara.Brasseur@oberlin.edu
}

\author{
Ryan E. Grady* \\ Department of Mathematics \\ University of Notre Dame \\ Notre Dame IN 46556 \\ rgrady@nd.edu
}

\author{
Stratos Prassidis* $\dagger$ \\ Department of Mathematics \\ Canisius College \\ Buffalo NY 14208 \\ prasside@canisius.edu
}

Submitted: Jun 27, 2007; Accepted: Feb 19, 2009; Published: Feb 27, 2009

Mathematics Subject Classification: 05C50 (60B15, 60J15)

\begin{abstract}
Combinatorial covers of graphs were defined by Chung and Yau. Their main feature is that the spectra of the Combinatorial Laplacian of the base and the total space are related. We extend their definition to directed graphs. As an application, we compute the spectrum of the Combinatorial Laplacian of the homesick random walk $R W_{\mu}$ on the line. Using this calculation, we show that the heat kernel on the weighted line can be computed from the heat kernel of ' $(1+1 / \mu)$-regular' tree.
\end{abstract}

\section{Introduction}

A finite presentation of a group determines a locally finite graph, the Cayley graph. In general, the graph depends on the presentation and it does not reflect the algebraic properties of the group. But the Cayley graph provides information on the "large scale" properties of the group i.e., properties of the group at infinity. This is the motivation for the question posed by M. Gromov:

\footnotetext{
*Partially Supported by an N.S.F. R.E.U. Grant

${ }^{\dagger}$ Partially Supported by a Canisus College Summer Research Grant
} 
Question. What is the relation between the spectrum of a random walk on the Cayley graph of the group to the geometry at infinity of the group?

A classical result towards this direction is given in [5] where the spectral radius (the maximal eigenvalue) of the normalized adjacency operator on a graph is connected to properties of the graph and the group. Notice that the normalized adjacency operator encodes the simple random walk on the graph, the one that each direction has the same probability.

One of the properties of the group that is reflected to its Cayley graph is the rate of growth of the elements of the group when they expresses as products of the generators and their inverses. The corresponding quantity in the Cayley graph is the rate of growth of the elements of the combinatorial spheres (or balls) centered at the vertex that represents the identity element. In [6], a family of random walks $R W_{\mu}$ are defined on a rooted graph. They are nearest neighbor "homesick" random walks that depend on a parameter $\mu$. In these random walks it is $\mu$ times more likely for the particle to move towards the root than to move away from it. The main result in [6] is that the growth of the graph is a threshold value. The random walk is transient if and only if $\mu$ is larger than the growth and positive recurrent if and only if $\mu$ is smaller than the growth. When the graph is the Cayley graph of a group, the result states that the growth of the group can be predicted from the properties of the homesick random walk on the Cayley graph.

The goal of this project is to explain Lyons' result using spectra of operators defined on the Hilbert space generated by the vertices of the graph. The definition of the random walk in [6] equips the graph with the structure of a directed weighted graph. The random walk in this case is encoded by the adjacency operator for this graph. Following the ideas in [1] and [3], we will work instead with a variant of the adjacency operator, the combinatorial Laplacian.

In [1] and [3], the definition of the combinatorial Laplacian is given. Also, graph coverings are used for the calculation of the spectrum and a basis of eigenfunctions of the Laplacian. The idea is that when a graph $G$ covers a graph $H$, then the combinatorial structure of $H$ is simpler and the Laplacian can be calculated for $H$. Then the authors develop methods to deduce from that Laplacian of $G$. The calculations are used for getting a closed formula for the heat kernel associated to the combinatorial Laplacian for $k$-regular trees (Cayley graphs of free groups and free products of copies of $\mathbb{Z} / 2 \mathbb{Z}$ ) and lattices (Cayley graphs of free abelian groups). In [2], the definition of the combinatorial Laplacian is extended to directed, weighted graphs and the basic properties of the construction are proved.

We extend the methods of [3] to weighted directed graphs. More specifically, we define combinatorial covers in this case. We extend the methods of [3] and use them to compare the eigenvalues and eigenfunctions of the base and the cover graphs. As an application, we calculate the heat kernel of the homesick random walk on the 2-regular tree. As in [3], the heat kernel of the infinite tree is computed as the limit of the heat kernel of the $l$-combinatorial neighborhood of the root, as $l$ goes to infinity. For the $l$-neighborhood, we construct a combinatorial cover for the 2-regular tree to the weighted, directed segment. The general theory implies that there are two types of eigenvalues for the Laplacian in 
question. The first type includes eigenvalues that admit eigenfunctions that do not vanish at the root. Those eigenvalues are induced from the directed segment. The eigenfunctions for the second type of the eigenvalues vanish at the root. After taking limits as $l$ goes to infinity, our main result computes the heat kernel in this case.

Theorem (Main Theorem). For the homesick random walk with parameter $\mu$ on the infinite line, the heat kernel $H_{t}(a, b)$ satisfies:

1. When $b=0$ :

$$
H_{t}(a, 0)=\frac{\mu \sqrt{2(\mu+1)}}{\pi} \int_{0}^{\pi} \frac{e^{-t\left(1-\frac{2 \sqrt{\mu}}{\mu+1} \cos x\right)} \sin x\left[\frac{1}{\mu} \sin (a+1) x-\sin (a-1) x\right]}{\mu^{2}+2 \mu+1-4 \mu \cos ^{2} x} d x .
$$

2. If both $a$ and $b$ are not 0 , then $H_{t}(a, b)$ is:

$$
K \int_{0}^{\pi} \frac{e^{-t\left(1-\frac{2 \sqrt{\mu}}{\mu+1} \cos x\right)}[\sin ((|a|+1) x)-\sin (|a|-1) x][\sin ((|b|+1) x)-\sin (|b|-1) x]}{\mu^{2}+2 \mu+1-4 \mu \cos ^{2} x} d x,
$$

where $K=\frac{2}{\pi}\left(\mu^{2}+\varepsilon\right)$ and $\varepsilon=\operatorname{sign}(a) \cdot \operatorname{sign}(b)$.

Notice that, up to a constant multiple, the formula (1) is the formula given in Theorem 2 in [3] for $k$ regular trees if we set $k=1+1 / \mu$. In some sense, the formula in (1) is the heat kernel on the $1+1 / \mu$-regular tree, even though $1+1 / \mu$ is not an integer.

Trees are (topological) covers of graphs. In this sense they are universal among graphs. The authors intend to extend the calculations to $k$-regular trees. The goal is to get a formulation of Lyons' theorem using the spectrum of the heat kernel on the Cayley graph. The authors believe that such a formulation will extend Lyons' result giving more information on the growth of the graph (or group).

The first two authors would like to thank Canisius College for its hospitality during the R.E.U. program in the summer 2006, when this paper was completed. All three authors would like to thank Terry Bisson for discussions during the preparation of this project.

\section{Preliminaries}

All graphs considered will be locally finite as undirected graphs i.e., only finitely many edges will be incident to a given vertex. We consider a weighted directed graph $G$ which has a vertex set $V=V(G)$ and a weight function $w: V \times V \rightarrow \mathbb{R}$ such that

$$
w(u, v) \geq 0, \text { for all } u, v \in V .
$$

For $u, v \in V$, if $w(u, v)>0$, then we say $(u, v)$ is an edge and $u$ is adjacent to $v$. The degree $d_{v}$ of a vertex $v$ is defined as:

$$
d_{v}=\sum_{u \in V} w(v, u)
$$


Our main example of weighted directed graphs will be the lazy random walks used in [6]. Given a simple undirected unweighted rooted graph $(G, z)$ we weight the edge $(u, v)$ as follows:

$$
w(u, v):= \begin{cases}\frac{\mu}{d_{u}+(\mu-1) d_{u}^{-}}, & v \in S_{|u|-1}(z) \\ \frac{1}{d_{u}+(\mu-1) d_{u}^{-}}, & \text {otherwise }\end{cases}
$$

where $d_{u}$ is the degree of $u$ and $d_{u}^{-}=\left|N(u) \cap S_{|u|-1}(z)\right|$, where $S_{r}(z)$ denotes the combinatorial ball of radius $r$ centered at $z$. That defines a random walk on $G$ denoted $R W_{\mu}$.

In [6], the properties of $R W_{\mu}$ on a Cayley graph are connected to the growth of the group. Let $\Gamma$ be a finitely presented group and $X$ a finite generating set. We assume that $X$ is symmetric i.e., $X=X^{-1}$. The Cayley graph, $\mathcal{G}(\Gamma, X)$ is the (non-directed) graph with vertex set $\Gamma$. Also, the pair $\left(\gamma_{1}, \gamma_{2}\right)$ is an edge if $\gamma_{1}^{-1} \gamma_{2} \in X$. Also, define the length of an element $\gamma \in \Gamma$ as

$$
\ell(\gamma)=\min \left\{r: \gamma=v_{1} \ldots v_{r}, \quad v_{i} \in X\right\} .
$$

Let $\sigma_{r}$ be the number of elements of $\Gamma$ of length $r$. Notice that $\sigma_{r}$ is the number of points on the combinatorial sphere of $\mathcal{G}(\Gamma, X)$ centered at the identity of radius $r$. The growth of the group is defined as $([4])$ :

$$
\omega(\Gamma, X)=\limsup _{r \rightarrow \infty} \sqrt[r]{\sigma_{r}}
$$

If $\omega(\Gamma, X)>1$ then $\Gamma$ is said to have exponential growth, if $\omega(\Gamma, X)=1$ the growth is subexponential and if $\sigma_{r}$ has the same growth as a polynomial function, $\Gamma$ is said to have polynomial growth. In [6], it was shown that:

- If $\mu<\omega(\Gamma, X)$, then $R W_{\mu}$ is transient.

- If $\mu>\omega(\Gamma, X)$, then $R W_{\mu}$ is positive recurrent

We will use covers to study the spectrum of a random walk.

Definition 2.1. Let $\widetilde{G}$ and $G$ be two weighted directed graphs. We say $\widetilde{G}$ is a covering of $G$ (or $G$ is covered by $\widetilde{G}$ ) if there is a map $\pi: V(\widetilde{G}) \rightarrow V(G)$ satisfying the following two properties:

(i) There is an $m \in \mathbb{R}$, called the index of $\pi$; such that for $u, v \in V(G)$, we have

$$
\sum_{\substack{x \in \pi^{-1}(u) \\ y \in \pi^{-1}(v)}} w(x, y)=m w(u, v), \text { and } \sum_{\substack{x \in \pi^{-1}(u) \\ y \in \pi^{-1}(v)}} w(y, x)=m w(v, u) .
$$

(ii) For $x, y \in V(\widetilde{G})$ with $\pi(x)=\pi(y)$ and $v \in V(G)$, we have

$$
\sum_{z \in \pi^{-1}(v)} w(z, x)=\sum_{z^{\prime} \in \pi^{-1}(v)} w\left(z^{\prime}, y\right), \text { and } \sum_{z \in \pi^{-1}(v)} w(x, z)=\sum_{z^{\prime} \in \pi^{-1}(v)} w\left(y, z^{\prime}\right) .
$$


Remark 2.2.

1. Notice that the definition generalizes the definition given in [3]. We need to consider "two sided" sums because the graphs are directed.

2. The definition of covering given above does not correspond to the classical definition of graph coverings, that is the direct generalization of graph coverings to directed weighted graphs. Let $\widetilde{G}$ and $G$ be directed, weighted graphs. A map $\pi: V(\widetilde{G}) \rightarrow$ $V(G)$ is called a classical graph covering if the following hold:

(a) For each $x, y$ in $V(\widetilde{G}), w(x, y)=w(\pi(x), \pi(y))$. Thus $\pi$ induces a map on the set of edges on $\widetilde{G}$.

(b) For each $x \in V(\widetilde{G})$ we write

$$
N^{\text {out }}(x)=\{e \in E(\widetilde{G}): e \text { emanates from } x\} .
$$

Then the map $\pi$ induces a bijection

$$
\pi: N^{\text {out }}(x) \rightarrow N^{\text {out }}(f(x))
$$

such that $w(e)=w(\pi(e))$.

In this paper, a cover will mean a covering as in the definition above.

As in [3], it is not hard to show the following properties of covers.

Lemma 2.3. Suppose $\widetilde{G}$ is a covering of $G$ with index $m$. Let $u, v \in V(G)$ and $x \in \pi^{-1}(v)$, then

(i)

$$
\sum_{z \in \pi^{-1}(u)} w(x, z)=\frac{m}{\left|\pi^{-1}(v)\right|} w(v, u)
$$

(ii)

$$
\sum_{z \in V(\widetilde{G})} w(x, z)=\frac{m}{\left|\pi^{-1}(v)\right|} \sum_{u \in V(G)} w(v, u)
$$

(iii)

$$
d_{x}=\frac{m}{\left|\pi^{-1}(v)\right|} d_{v}
$$

From the definition of a covering (i) and (ii) still hold if we replace $w(x, z)$ by $w(z, x)$ and $w(v, u)$ by $w(u, v)$. 
Let $G$ be a finite graph. The transition probability matrix $P$ of a graph is defined as

$$
P(u, v)=\frac{w(u, v)}{d_{u}} .
$$

It is obvious that $P(u, v)>0$ only if $(u, v)$ is an edge. Further as in the undirected case

$$
\sum_{v} P(u, v)=1
$$

however in the directed case it is not true in general that $\sum_{u} P(u, v)=1$. The transition probability matrix defines a random walk on the graph.

The Perron-Frobenius Theorem ([7]) implies that the transition probability matrix $P$ of a graph has a unique left eigenvector $\phi$ with $\phi(v)>0$ for all $v$, and $\phi P=\phi$. We will treat $\phi$ as a row vector. We can normalize and choose $\phi$ such that

$$
\sum_{v} \phi(v)=1
$$

We call $\phi$ the Perron vector of $P$. We can now define the Laplacian $\mathcal{L}$ of a directed graph $([2])$ :

$$
\mathcal{L}=I-\frac{\Phi^{1 / 2} P \Phi^{-1 / 2}+\Phi^{-1 / 2} P^{*} \Phi^{1 / 2}}{2}
$$

where $\Phi$ denotes the diagonal matrix with entries $\Phi(v, v)=\phi(v)$ and $P^{*}$ is the transpose of $P$. The Laplacian satisfies $\mathcal{L}^{*}=\mathcal{L}$, that is, the Laplacian is symmetric. The spectrum of a graph is the eigenvalues and eigenfunctions of the Laplacian of the graph.

We give an extension of the definition of the combinatorial Laplacian to infinite rooted graphs. Let $(G, z)$ be any locally finite weighted directed graph. Let $B_{r}(z)$ be the ball of radius $r$ centered at $z$ when $G$ is considered as a simple graph i.e., when the weights and the orientation are ignored. Then for $u, v \in V(G)$ define

$$
\mathcal{L}(u, v)=\mathcal{L}^{(l)}(u, v)
$$

where $\mathcal{L}^{(l)}$ is the combinatorial Laplacian of $B_{l}(z)$ with $l$ large enough so that $u$ and $v$ are contained in $B_{l-2}(z)$. Since $\mathcal{L}^{(l)}$ depends on the nearest neighbors, the definition of $\mathcal{L}$ does not depend on $l$. It is a direct calculation that $l$ defines an operator on $L^{2}(V(G))$ when there is a uniform bound on the degrees $d_{v}$, with $v \in V(G)$.

\subsection{Example - The $k$-regular Tree}

Consider the homesick random walk on the $k$-regular tree $T_{k}$ with root $z$. In particular the weights are given as follows:

$$
w(x, y)=\left\{\begin{aligned}
\frac{1}{k}, & x=z, y \sim z, \\
\frac{1}{k+\mu-1}, & x \neq z, y \notin S_{|x|-1}(z), \\
\frac{\mu}{k+\mu-1}, & x \neq z, y \in S_{|x|-1}(z)
\end{aligned}\right.
$$


The combinatorial $l$-sphere centered at $z$ has $s_{l}=k(k-1)^{l-1}$ if $l>0$. We write

$$
v_{i}^{(l)}, \quad i=1,2, \cdots, s_{l},
$$

for the elements of the combinatorial $l$-sphere centered at $z$. Let $T_{k}^{(l)}$ be the $l$-ball in $T_{k}$ i.e., we truncate $T_{k}$ to include all the vertices within combinatorial distance $l$ from $z$. We consider the homesick random walk induced on $T_{k}^{(l)}$. It has the same weights as the random walk on $T_{k}$ except that:

$$
w\left(v_{i}^{(l)}, v\right)=1 \text {, when } v_{i}^{(l)} \sim v
$$

Let $P^{(l)}$ be the matrix of the random walk. Also, set

$$
\rho=\frac{k-1}{\mu} .
$$

Lemma 2.4. Let $\psi_{l}$ be the Frobenius-Perron vector of $T_{k}^{(l)}$. Then

$$
\psi_{l}\left(v_{i}^{(m)}\right)=\psi_{l}\left(v_{i}^{(m)}\right), \text { for all } 0 \leq m \leq l, 1 \leq i, j \leq s_{m} .
$$

Thus $\psi_{l}$ is constant along each sphere centered at z. Furthermore,

$$
\psi_{l}\left(v_{i}^{(n)}\right)= \begin{cases}\frac{1-\rho}{2\left(1-\rho^{l}\right)}, & n=0 \\ \frac{(1+\rho)(1-\rho)}{2 \mu^{n-1} k\left(1-\rho^{l}\right)}, & 1 \leq n \leq l-1 \\ \frac{1-\rho}{2 \mu^{l-1} k\left(1-\rho^{l}\right)}, & n=l .\end{cases}
$$

Proof. In the process of calculating the Frobenius-Perron eigenfunction we will show that its values are constant on the spheres of $T_{k}^{(l)}$. For simplicity, we set:

$$
\alpha=\frac{\mu}{k+\mu-1}, \quad \beta=\frac{1}{k+\mu-1}
$$

For any $v_{i}^{(l)}$ there is a single vertex $v_{j}^{(l-1)}$ that is adjacent to it. The equation $\psi P^{(l)}=\psi$ implies

$$
\beta \psi\left(v_{j}^{(l-1)}\right)=\psi\left(v_{i}^{(l)}\right) \text { for each } v_{i}^{(l)} \sim v_{j}^{(l-1)} .
$$

For a vertex $v_{j}^{(l-1)}$, using the previous equation,

$$
\beta \psi\left(v_{m}^{(l-2)}\right)+(k-1) \psi\left(v_{i}^{(l)}\right)=\psi\left(v_{j}^{(l-1)}\right), \quad v_{m}^{(l-2)} \sim v_{j}^{(l-1)}, v_{i}^{(l)} \sim v_{j}^{(l-1)} .
$$

That implies

$$
\beta \psi\left(v_{m}^{(l-2)}\right)=(1-\beta(k-1)) \psi\left(v_{j}^{(l-1)}\right) .
$$


Solving for $\psi\left(v_{j}^{(l-1)}\right)$, we get

$$
\psi\left(v_{j}^{(l-1)}\right)=\frac{1}{\mu} \psi\left(v_{m}^{(l-2)}\right) .
$$

Thus for two vertices in $S_{l-1}$ have a common neighbor in $S_{l-2}$ then $\psi$ has the same value. For the next step notice that

$$
\beta \psi\left(v_{n}^{(l-3)}\right)+(k-1) \alpha \psi\left(v_{j}^{(l-1)}\right)=\psi\left(v_{m}^{(l-2)}\right), \quad v_{n}^{(l-3)} \sim v_{m}^{(l-2)}, v_{j}^{(l-1)} \sim v_{m}^{(l-2)} .
$$

As before simplifying,

$$
\psi\left(v_{m}^{(l-2)}\right)=\frac{1}{\mu} \psi\left(v_{n}^{(l-3)}\right)
$$

Continuing like that we get that

$$
\psi\left(v_{m}^{(t)}\right)=\frac{1}{\mu} \psi\left(v_{n}^{(t-1)}\right), \quad v_{m}^{(t)} \sim v_{n}^{(t-1)}, \quad l-2 \leq t \leq 2 .
$$

For the vertices of distance 1 from $z$,

$$
\frac{1}{k} \psi(z)+(k-1) \alpha \psi\left(v_{i}^{(2)}\right)=\psi\left(v_{j}^{(1)}\right), \quad v_{i}^{(2)} \sim v_{j}^{(1)} .
$$

Thus

$$
\frac{1}{k} \psi(z)=\left(1-\frac{(k-1) \alpha}{\mu}\right) \psi\left(v_{j}^{(1)}\right) \Longrightarrow \psi\left(v_{j}^{(1)}\right)=\frac{1}{k \alpha} \psi(z)=\frac{k+\mu-1}{\mu k} \psi(z)
$$

Therefore $\psi$ has the same value on $S_{1}$ and thus $\psi$ is constant on each sphere. Inductively, we get that

$$
\psi_{l}\left(v_{i}^{(n)}\right)=\left\{\begin{array}{ll}
\frac{k+\mu-1}{\mu^{n} k} \psi_{l}(z), & 1 \leq n \leq l-1 \\
\frac{1}{\mu^{l-1} k} \psi_{l}(z), & n=l .
\end{array}= \begin{cases}\frac{1+\rho}{\mu^{n-1} k} \psi_{l}(z), & 1 \leq n \leq l-1 \\
\frac{1}{\mu^{l-1} k} \psi_{l}(z), & n=l .\end{cases}\right.
$$

Adding up all the terms:

$$
\begin{aligned}
\sum_{i, n} \psi_{l}\left(v_{i}^{(n)}\right) & =\left(\frac{\mu+k-1}{\mu} \sum_{i=0}^{l-2} \rho^{i}+\rho^{l-1}+1\right) \psi_{l}(z) \\
& \left.=\left(\frac{(\rho+1)\left(1-\rho^{l-1}\right)}{1-\rho}+\rho^{l-1}+1\right)\right) \psi_{l}(z) \\
& =\left(\frac{2\left(1-\rho^{l}\right)}{1-\rho}\right) \psi_{l}(z)
\end{aligned}
$$

So the normalization condition implies:

$$
\psi_{l}(z)=\frac{1-\rho}{2\left(1-\rho^{l}\right)},
$$


and the Frobenius-Perron vector is given by:

$$
\psi_{l}\left(v_{i}^{(n)}\right)= \begin{cases}\frac{1-\rho}{2\left(1-\rho^{l}\right)}, & n=0 \\ \frac{(1+\rho)(1-\rho)}{2 \mu^{n-1} k\left(1-\rho^{l}\right)}, & 1 \leq n \leq l-1 \\ \frac{1-\rho}{2 \mu^{l-1} k\left(1-\rho^{l}\right)}, & n=l\end{cases}
$$

Remark 2.5. Notice that the limit, as $l \rightarrow \infty$ of $\psi_{l}(z)$ is non-zero if and only if $\rho<1$. That happens if and only if the random walk is positive recurrent ([6]). If this is the case,

$$
\psi(z)=\lim _{l \rightarrow \infty} \psi_{l}(z)=\frac{1-\rho}{2} .
$$

Furthermore, taking limits as $l \rightarrow \infty$, we get the following formula for a candidate for the Frobenius-Perron vector for the homesick random walk on $T_{k}$ :

$$
\psi_{l}\left(v_{i}^{(n)}\right)= \begin{cases}\frac{1-\rho}{2}, & n=0 \\ \frac{(1+\rho)(1-\rho)}{2 \mu^{n-1} k}, & n \geq 1 .\end{cases}
$$

A direct calculation shows that $\psi$ is the Frobenius-Perron vector for $T_{k}$, when $\rho<1$.

Let $\Psi_{l}$ be the diagonal matrix with entries $\psi_{l}\left(v_{i}^{(n)}\right)$. Then, for two adjacent vertices,

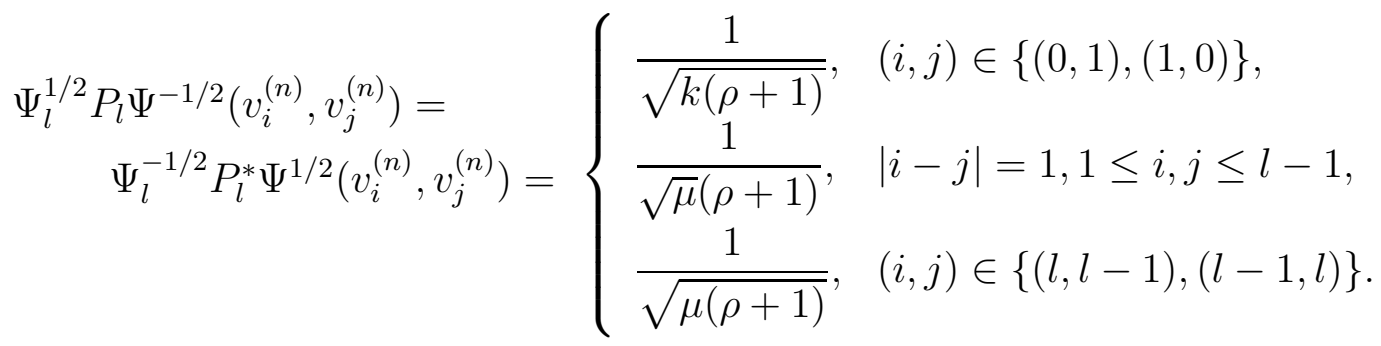

The combinatorial Laplacian on $T_{k}^{(l)}$ is given by:

$$
L^{(l)}\left(v_{i}^{(n)}, v_{j}^{(m)}\right)= \begin{cases}1, & v_{i}^{(n)}=v_{j}^{(m)}, \\ -\frac{1}{\sqrt{k(\rho+1)},} & (n, m) \in\{(0,1),(1,0)\}, \\ -\frac{1}{\sqrt{\mu}(\rho+1)}, & |n-m|=1,1 \leq n, m \leq l-1, v_{i}^{(n)} \sim v_{j}^{(m)} \\ -\frac{1}{\sqrt{\mu(\rho+1)},} & (n, m) \in\{(l, l-1),(l-1, l)\}, \\ 0, & \text { if the vertices are not adjacent. }\end{cases}
$$




\section{The spectrum of a graph and its coverings}

We will show that there is a connection between the eigenvalues of a graph and a graph that covers it, however to begin we establish a connection between the respective Perron vectors. All the graphs are finite.

Proposition 3.1. Suppose $G$ is a weighted directed graph with Perron vector $\phi$ and $\widetilde{G}$ is a covering of $G$ with index $m$ with respect to the mapping $\pi$. The Perron vector $\tilde{\phi}$ of $\widetilde{G}$ can be defined by

$$
\tilde{\phi}(x)=\frac{\phi(v)}{\left|\pi^{-1}(v)\right|}, \text { for each } v=\pi(x) \text {. }
$$

Proof. It is enough to show that $\tilde{\phi} \tilde{P}=\tilde{\phi}$, where $\tilde{P}$ is the transition probability matrix of $\widetilde{G}$. We make liberal use of Lemma 2.3. Suppose $v \in V(G)$ and $\pi(x)=v$. Then

$$
(\tilde{\phi} \tilde{P})(x)=\sum_{y \in \widetilde{G}} \tilde{\phi}(y) \tilde{P}(y, x)=\sum_{u \in V(G)} \sum_{y \in \pi^{-1}(u)} \tilde{\phi}(y) \tilde{P}(y, x)
$$

By substituting the values of $\tilde{\phi}$ and $\tilde{P}$, the last equation becomes

$$
(\tilde{\phi} \tilde{P})(x)=\sum_{u \in V(G)} \sum_{y \in \pi^{-1}(u)} \frac{\phi(u)}{\left|\pi^{-1}(u)\right|} \frac{w(y, x)}{d_{y}}=\sum_{u \in V(G)} \frac{\phi(u)}{\left|\pi^{-1}(u)\right|} \sum_{y \in \pi^{-1}(u)} \frac{\left|\pi^{-1}(u)\right|}{m d_{u}} w(y, x)
$$

By rearranging the terms we get that

$$
(\tilde{\phi} \tilde{P})(x)=\sum_{u \in V(G)} \frac{\phi(u)}{m d_{u}} \sum_{y \in \pi^{-1}(u)} w(y, x)=\sum_{u \in V(G)} \frac{\phi(u)}{m d_{u}} \frac{m}{\left|\pi^{-1}(x)\right|} w(u, v)
$$

Using the definitions, once more

$$
(\tilde{\phi} \tilde{P})(x)=\frac{1}{\left|\pi^{-1}(v)\right|} \sum_{u \in V(G)} \phi(u) \frac{w(u, v)}{d_{u}}=\frac{1}{\left|\pi^{-1}(v)\right|} \sum_{u \in V(G)} \phi(u) P(u, v)=\frac{1}{\left|\pi^{-1}(v)\right|} \phi(v)
$$

Since $\phi$ is an eigenfunction, we get that:

$$
(\tilde{\phi} \tilde{P})(x)=\frac{1}{\left|\pi^{-1}(v)\right|}(\phi P)(v)=\frac{1}{\left|\pi^{-1}(v)\right|} \phi(v)=m \tilde{\phi}(x) .
$$

Thus $\tilde{\phi}$, as defined in the statement of the Proposition, is the Perron vector of $\widetilde{G}$.

When computing the spectrum of a graph it is sometimes convenient to consider harmonic eigenfunctions. Let $g$ denote an eigenfunction of $\mathcal{L}$ associated with the eigenvalue $\lambda$ then $f=g \Phi^{-1 / 2}$ is called the harmonic eigenfunction. In [2], it was shown that:

$$
\lambda f(v) \phi(v)=\frac{1}{2} \sum_{u}(f(v)-f(u))(\phi(u) P(u, v)+P(v, u) \phi(v)), \text { for each } v \in V(G),
$$

where $f$ is a harmonic eigenfunction associated with the eigenvalue $\lambda$.

As in [3], using the above identity we get the following: 
Lemma 3.2. If $\widetilde{G}$ is a covering of $G$, then an eigenvalue of $G$ is an eigenvalue of $\widetilde{G}$.

Proof. If $\widetilde{G}$ is a covering of $G$ with respect to the map $\pi$ of index $m$, we can lift the harmonic eigenfunction $f$ of $G$ (and the associated eigenvalue $\lambda$ ) to $\widetilde{G}$ by defining, for each vertex $x$ in $\widetilde{G}, f(x)=f(v)$ where $v=\pi(x)$. We then have

$$
\begin{aligned}
\frac{1}{2} \sum_{y}( & f(x)-f(y))(\tilde{\phi}(y) \tilde{P}(y, x)+\tilde{P}(x, y) \tilde{\phi}(x)) \\
= & \frac{1}{2} \sum_{y}(f(x)-f(y))\left(\tilde{\phi}(y) \frac{w(y, x)}{d_{y}}+\frac{w(x, y)}{d_{x}} \tilde{\phi}(x)\right) \\
= & \frac{1}{2} \sum_{u} \sum_{z \in \pi^{-1}(u)}(f(v)-f(u))\left(\frac{\phi(u)}{\left|\pi^{-1}(u)\right|} \frac{w(z, x)}{d_{z}}+\frac{w(x, z)}{d_{x}} \frac{\phi(v)}{\left|\pi^{-1}(v)\right|}\right) \\
= & \frac{1}{2} \sum_{u}(f(v)-f(u))\left(\frac{\phi(u)}{\left|\pi^{-1}(u)\right|} \sum_{z \in \pi^{-1}(u)} \frac{w(z, x)}{d_{z}}+\frac{\phi(v)}{\left|\pi^{-1}(v)\right|} \sum_{z \in \pi^{-1}(u)} \frac{w(x, z)}{d_{x}}\right) \\
= & \frac{1}{2} \sum_{u}(f(v)-f(u))\left(\frac{\phi(u)}{\left|\pi^{-1}(u)\right|} \sum_{z \in \pi^{-1}(u)} \frac{\left|\pi^{-1}(u)\right| w(z, x)}{m d_{u}}+\frac{\phi(v)}{\left|\pi^{-1}(v)\right|} \sum_{z \in \pi^{-1}(u)} \frac{\left|\pi^{-1}(v)\right| w(x, z)}{m d_{v}}\right) \\
= & \frac{1}{2} \sum_{u}(f(v)-f(u))\left(\frac{\phi(u)}{m d_{u}} \sum_{z \in \pi^{-1}(u)} w(z, x)+\frac{\phi(v)}{m d_{v}} \sum_{z \in \pi^{-1}(u)} w(x, z)\right) \\
= & \frac{1}{2} \sum_{u}(f(v)-f(u))\left(\frac{\phi(u)}{m d_{u}} \frac{m}{\left|\pi^{-1}(v)\right|} w(u, v)+\frac{\phi(v)}{m d_{v}} \frac{m}{\left|\pi^{-1}(v)\right|} w(v, u)\right) \\
= & \frac{1}{\left|\pi^{-1}(v)\right|} \frac{1}{2} \sum_{u}(f(v)-f(u))(\phi(u) P(u, v)+P(v, u) \phi(v)) \\
= & \frac{1}{\left|\pi^{-1}(v)\right|} \lambda f(v) \phi(v)=\lambda f(x) \tilde{\phi}(x) .
\end{aligned}
$$

Following the line of argument in [3], we have the following:

Lemma 3.3. Suppose $\widetilde{G}$ is a covering of $G$ with respect to the mapping $\pi$ of index $m$. If a harmonic eigenfunction $f$ of $\widetilde{G}$, associated with an eigenvalue $\lambda$, has a nontrivial image in $G$, then $\lambda$ is also an eigenvalue for $G$.

Proof. For each $x \in \pi^{-1}(v)$,

$$
\sum_{y}(f(x)-f(y))(\tilde{\phi}(y) \tilde{P}(y, x)+\tilde{P}(x, y) \tilde{\phi}(x))=\lambda f(x) \tilde{\phi}(x) .
$$

By summing over $x$ in $\pi^{-1}(v)$, we have

$$
\sum_{x \in \pi^{-1}(v)} \sum_{y}(f(x)-f(y))(\tilde{\phi}(y) \tilde{P}(y, x)+\tilde{P}(x, y) \tilde{\phi}(x))=\lambda \sum_{x \in \pi^{-1}(v)} f(x) \tilde{\phi}(x) .
$$

We define the induced mapping of $f$ in $G$, denoted $g: V(G) \rightarrow \mathbb{R}$ by

$$
g(v)=\sum_{x \in \pi^{-1}(v)} \frac{f(x) \tilde{\phi}(x)}{\phi(v)}=\frac{1}{\left|\pi^{-1}(v)\right|} \sum_{x \in \pi^{-1}(v)} f(x) .
$$


It is clear from the definition of $g$ that

$$
\lambda \sum_{x \in \pi^{-1}(v)} f(x) \tilde{\phi}(x)=\lambda g(v) \phi(v) .
$$

Now consider the following

$$
\frac{1}{2} \sum_{x \in \pi^{-1}(v)} \sum_{y}(f(x)-f(y))(\tilde{\phi}(y) \tilde{P}(y, x)+\tilde{P}(x, y) \tilde{\phi}(x)) .
$$

We break the sum into two parts:

(i)

$$
\begin{aligned}
\frac{1}{2} \sum_{x \in \pi^{-1}(v)} & f(x) \sum_{y}(\tilde{\phi}(y) \tilde{P}(y, x)+\tilde{P}(x, y) \tilde{\phi}(x)) \\
= & \frac{1}{2} \sum_{x \in \pi^{-1}(v)} f(x)(\tilde{\phi}(x)+\tilde{\phi}(x)) \\
= & \sum_{x \in \pi^{-1}(v)} f(x) \tilde{\phi}(x) \\
= & \frac{1}{2} g(v)(\phi(v)+\phi(v)) \\
= & \frac{1}{2} \sum_{u} g(v)(\phi(u) P(u, v)+P(v, u) \phi(v)) .
\end{aligned}
$$

(ii)

$$
\begin{aligned}
\frac{1}{2} \sum_{x \in \pi^{-1}(v)} \sum_{y} f(y)(\tilde{\phi}(y) \tilde{P}(y, x)+\tilde{P}(x, y) \tilde{\phi}(x)) \\
=\frac{1}{2} \sum_{u} \sum_{y \in \pi^{-1}(u)} f(y) \sum_{x \in \pi^{-1}(v)}(\tilde{\phi}(y) \tilde{P}(y, x)+\tilde{P}(x, y) \tilde{\phi}(x)) \\
=\frac{1}{2} \sum_{u} \sum_{y \in \pi^{-1}(u)} f(y) \sum_{x \in \pi^{-1}(v)}\left(\frac{\phi(u) w(y, x)}{\left|\pi^{-1}(u)\right| d_{y}}+\frac{w(x, y) \phi(v)}{d_{x}\left|\pi^{-1}(v)\right|}\right) \\
=\frac{1}{2} \sum_{u} \sum_{y \in \pi^{-1}(u)} f(y)\left(\frac{\phi(u)}{m d_{u}} \sum_{x \in \pi^{-1}(v)} w(y, x)+\frac{\phi(v)}{m d_{v}} \sum_{x \in \pi^{-1}(v)} w(x, y)\right) \\
=\frac{1}{2} \sum_{u} \sum_{y \in \pi^{-1}(u)} f(y)\left(\frac{\phi(u) w(u, v)}{d_{u}\left|\pi^{-1}(u)\right|}+\frac{\phi(v) w(v, u)}{d_{v}\left|\pi^{-1}(u)\right|}\right) \\
=\frac{1}{2} \sum_{u} g(u)(\phi(u) P(u, v)+P(v, u) \phi(v)) .
\end{aligned}
$$


We can now write expression (1) as

$$
\frac{1}{2} \sum_{u}(g(v)-g(u))(\phi(u) P(u, v)+P(v, u) \phi(u)) .
$$

Hence,

$$
\frac{1}{2} \sum_{u}(g(v)-g(u))(\phi(u) P(u, v)+P(v, u) \phi(u))=\lambda g(v) \phi(v) .
$$

If $g$ is nontrivial, then $\lambda$ is an eigenvalue of $G$.

Definition 3.4. A graph $\widetilde{G}$ is a regular covering of $G$ if for a fixed vertex $v$ in $V(G)$ and for any vertex $x$ of $V(\widetilde{G}), \widetilde{G}$ is a covering of $G$ under a mapping $\pi_{x}$ which maps $x$ into $v$. If $\pi_{x}^{-1}$ is just $x$ then $\widetilde{G}$ is a strong regular covering. Further, a graph $G$ is said to be distance regular if $G$ is a strong regular covering of a (weighted) path.

Remark 3.5. Strong regular coverings do not appear as often in weighted directed graphs. The reason is that the definition requires some degree of homogeneity. Usually, this type of homogeneity is lacking in the lazy random walks considered.

Lemma 3.6. Suppose $\widetilde{G}$ is a strong regular covering of $G$. Then, $\widetilde{G}$ and $G$ have the same eigenvalues.

Proof. For any nontrivial harmonic eigenfunction $f$ of $\widetilde{G}$ we can choose $v$ to be a vertex with nonzero value of $f$. The induced mapping of $f$ in $G$ has a nonzero value at $v$ and therefore is a nontrivial harmonic eigenfunction for $G$. From Lemma 3.3, we see that any eigenvalue of $\widetilde{G}$ is an eigenvalue of $G$. By Lemma 3.2, we conclude that $\widetilde{G}$ and $G$ have the same eigenvalues.

\subsection{Example - The $k$-regular Tree as a Cover}

As in [3], we will realize $T_{k}$ as a cover over a weighted ray. Let $\mathcal{P}^{+}$be the weighted ray with $V\left(\mathcal{P}^{+}\right)=\mathbb{N}$ and

$$
w(i, j)= \begin{cases}1, & \text { if } i=0, j=1 \\ \frac{k \mu(k-1)^{i-1}}{\mu+k-1}, & \text { if } i>0, i-j=1 \\ \frac{k(k-1)^{i}}{\mu+k-1}, & \text { if } i>0, i-j=-1\end{cases}
$$

As in [3],

$$
\pi: T_{k} \rightarrow \mathcal{P}^{+}, \pi(x)=d(x, z),
$$

where the distance is the combinatorial distance in $T_{k}$. The fact that $\pi$ is a combinatorial cover of index 1 is proved as in [3]. 
This means that some of the eigenvalues of the tree are the same as those of the path. Thus we set out to determine the eigenvalues and eigenfunctions of the path.

First we must find the Laplacian of $\mathcal{P}^{+}$. To do that, let $\mathcal{P}^{(l)}$ be truncated path with

$$
V\left(\mathcal{P}^{(l)}\right)=\{0,1, \ldots, l\}
$$

and all the weights as in $\mathcal{P}^{+}$except that we set $w(l, l-1)=1$. We will calculate the Laplacian of $\mathcal{P}^{(l)}$ and then we will take $l \rightarrow \infty$. The probability matrix is given by:

$$
P_{l}=\left(\begin{array}{ccccc}
0 & 1 & 0 & \cdots & \\
\frac{1}{1+\rho} & 0 & \frac{\rho}{1+\rho} & \cdots & \\
0 & \frac{1}{1+\rho} & 0 & \frac{\rho}{1+\rho} & \\
\vdots & \ddots & \ddots & \ddots & \vdots \\
0 & \cdots & 0 & 1 & 0
\end{array}\right)
$$

The Frobenius-Perron vector satisfies

$$
\left(\phi_{l}(0), \phi_{l}(1), \phi_{l}(2), \ldots, \phi_{l}(l)\right) P_{l}=\left(\phi_{l}(0), \phi_{l}(1), \phi_{l}(2), \ldots, \phi_{l}(l)\right)
$$

Thus the vector $\phi_{l}$ satisfies:

$$
\begin{gathered}
\phi_{l}(i)= \begin{cases}\rho^{i-1}(1+\rho) \phi_{l}(0), & 1 \leq i \leq l-1 \\
\rho^{l-1} \phi_{l}(0), & i=l .\end{cases} \\
\Phi_{l}^{\frac{1}{2}} P_{l} \Phi_{l}^{-\frac{1}{2}}(i, j)=\Phi_{l}^{-\frac{1}{2}} P_{l}^{*} \Phi_{l}^{\frac{1}{2}}(i, j)=\left\{\begin{aligned}
\frac{1}{\sqrt{1+\rho}}, \quad(i, j)=(0,1),(1,0) \\
\frac{\sqrt{\rho}}{1+\rho}, \quad|i-j|=1,2 \leq i \leq l-2 \\
\frac{\sqrt{\rho}}{1+\rho}, \quad(i, j)=(1,2),(l-1, l-2) \\
\sqrt{\frac{\rho}{1+\rho},} \quad(i, j)=(l-1, l),(l, l-1)
\end{aligned}\right.
\end{gathered}
$$

Hence,

$$
\mathcal{L}^{(l)}(i, j)=\left\{\begin{array}{cl}
1, & i=j \\
-\frac{1}{\sqrt{1+\rho}}, & (i, j)=(0,1),(1,0) \\
-\frac{\sqrt{\rho}}{1+\rho}, & |i-j|=1,2 \leq i \leq l-2 \\
-\frac{\sqrt{\rho}}{1+\rho}, & (i, j)=(1,2),(l-1, l-2) \\
-\sqrt{\frac{\rho}{1+\rho}}, & (i, j)=(l-1, l),(l, l-1)
\end{array}\right.
$$


So if we set $\sigma=1+\rho$, then we get:

$$
\mathcal{L}^{(l)}(i, j)=\left\{\begin{aligned}
1 ; & i=j, \\
-\sqrt{\frac{1}{\sigma}} ; & (i, j)=(0,1) \text { or }(1,0), \\
-\frac{\sqrt{\sigma-1}}{\sigma} ; & |i-j|=1,0<i, j<l, \\
-\sqrt{\frac{\sigma-1}{\sigma} ;} & (i, j)=(l-1, l) \text { or }(l, l-1), \\
0 ; & \text { otherwise. }
\end{aligned}\right.
$$

Note that this is exactly the same Laplacian as in [3] Section 5. Hence, the eigenvalues of $\mathcal{L}_{l}$ are 0,2 and

$$
\lambda_{n}=1-\frac{2 \sqrt{\sigma-1}}{\sigma} \cos \frac{\pi n}{l}=1-\frac{2 \sqrt{\mu(k-1)}}{\mu+k-1} \cos \frac{\pi n}{l}, \text { for } n=1, \ldots, l-1 .
$$

The eigenfunction $\phi_{0}$ associated with the eigenvalue 0 can be written as $f_{0} /\left\|f_{0}\right\|$ where

$$
\begin{aligned}
& f_{0}(0)=1 \\
& f_{0}(p)=\sqrt{\sigma(\sigma-1)^{p-1}}, \text { for } 1 \leq p \leq l-1, \\
& f_{0}(l)=\sqrt{(\sigma-1)^{l-1}} .
\end{aligned}
$$

We now consider the eigenfunction $\phi_{l}$ which corresponds to the eigenvalue 2 . In this case, $\phi_{l}=f_{l} /\left\|f_{l}\right\|$, where $f_{l}$ is defined as follows:

$$
\begin{aligned}
& f_{l}(0)=1 \\
& f_{l}(p)=(-1)^{p} \sqrt{\sigma(\sigma-1)^{p-1}}, \text { for } 1 \leq p \leq l-1, \\
& f_{l}(l)=(-1)^{l} \sqrt{(\sigma-1)^{l-1}} .
\end{aligned}
$$

For each $n \in\{1,2, \ldots, l-1\}$ there is an eigenfunction $\phi_{n}$ associated with the eigenvalue $\mu_{n}$. We can write $\phi_{n}=f_{n} /\left\|f_{n}\right\|$ where

$$
\begin{aligned}
f_{n}(0) & =\sqrt{\frac{\sigma}{\sigma-1}} \sin \frac{\pi n}{l} \\
f_{n}(p) & =\sin \frac{\pi n(p+1)}{l}-\frac{1}{\sigma-1} \sin \frac{\pi n(p-1)}{l}, \text { for } 1 \leq p \leq l-1, \\
f_{n}(l) & =\frac{\sqrt{\sigma}}{\sigma-1} \sin \frac{\pi n}{l} .
\end{aligned}
$$


For $n=1, \ldots, l-1$,

$$
\left\|f_{j}\right\|^{2}=\frac{l \sigma^{2}}{2(\sigma-1)^{2}}\left(1-\frac{4(\sigma-1)}{\sigma^{2}} \cos ^{2} \frac{n \pi}{l}\right) .
$$

The eigenfunction $\phi_{n} \in L^{2}\left(\mathcal{P}_{+}^{(l)}\right)$ for $n=0,1 \ldots l$.

Let $L^{2}\left(T_{k}^{(l)}\right)$ be the $L^{2}$-space generated by the vertices of $T_{k}^{(l)}$. Then

$$
L^{2}\left(T_{k}^{(l)}\right)=V_{1} \oplus V_{2}
$$

where is the direct sum of eigenspaces of the Laplacian $L^{(l)}$ on $T_{k}^{(l)}$ that contain eigenfunctions that not vanish at $z$. The second summand is its complementary subspace i.e., the subspace containing the eigenspaces that all eigenfunctions vanish at $z$. Let $\phi \in V_{1}$. If $s_{n}$ demote the number of elements in the combinatorial sphere of radius $n$ centered at $z$, Lemma 3.3 implies that the function

$$
g(n)=\frac{1}{s_{n}} \sum_{j=1}^{s_{n}} \phi\left(v_{j}^{(n)}\right)
$$

is an eigenfunction in of $\mathcal{L}^{(l)}$. But Lemma 2.4 implies that the values of $\phi$ at a vertex of $T_{k}^{(l)}$ depend only on the distance of the vertex from $z$. Thus the function $g(n)=\phi\left(v_{j}^{(n)}\right)$ is an eigenfunction of $\mathcal{L}^{(l)}$. Thus the eigenfunctions in $V_{1}$ are lifts of the eigenfunctions of the Laplacian $\mathcal{L}^{(l)}$.

\section{The heat kernel of a graph and its covering}

We extend the definition of the heat kernel ([1]) to directed graphs. Given a weighted directed graph $G$, the heat kernel $h_{t}$ is defined for $t \geq 0$. It is the solution to the heat equation:

$$
\frac{\partial h_{t}}{\partial t}=-\mathcal{L} h_{t}, \quad h_{0}=I
$$

Then $h_{t}$ can be expressed as

$$
h_{t}=e^{-t \mathcal{L}}=\sum_{r=0}^{\infty}(-1)^{r} \frac{t^{r} \mathcal{L}^{r}}{r !}=\sum_{i} e^{-\lambda_{i} t} P_{i}
$$

where $P_{i}$ is the projection into the eigenspace corresponding to the eigenvalue $\lambda_{i}$ of $\mathcal{L}$. If $u, v$ are vertices of $G$, then

$$
h_{t}(u, v)=\sum_{i} e^{-\lambda_{i} t} \psi_{i}(u) \psi_{i}(v)
$$

where $\psi_{i}$ ranges over the orthonormal eigenfunctions of $\mathcal{L}([1],[3])$. The definition of the Laplacian of the infinite graph implies that the heat kernel of the graph satisfies:

$$
h_{t}=\lim _{l \rightarrow \infty} h_{t}^{(l)}
$$


where $h_{t}^{(l)}$ is the heat kernel on $B_{l}(z)$.

For finite graphs, the eigenvalues of a graph $G$ and $\widetilde{G}$ a covering of $G$ are related. Therefore, it should be expected that their respective heat kernels are related. Indeed they are as illustrated by the following lemmas, which are the directed analogues of the results in [3]. Since the results and their proofs take place in finite balls around the vertices, they can be extended to infinite graphs.

An $r$-walk on a graph is a sequence of vertices $p_{r}=\left(u_{0}, u_{1}, \ldots, u_{r}\right)$ so that $\left(u_{i}, u_{i+1}\right)$ is an edge. The weight of $p_{r}$ is defined as

$$
\begin{aligned}
\omega\left(p_{r}\right) & =\prod_{i=0}^{r-1} \frac{\left(\Phi^{1 / 2} P \Phi^{-1 / 2}+\Phi^{-1 / 2} P^{*} \Phi^{1 / 2}\right)\left(u_{i}, u_{i+1}\right)}{2} \\
& =\prod_{i=0}^{r-1} \frac{\phi\left(u_{i}\right) P\left(u_{i}, u_{i+1}\right)+\phi\left(u_{i+1}\right) P\left(u_{i+1}, u_{i}\right)}{2 \sqrt{\phi\left(u_{i}\right) \phi\left(u_{i+1}\right)}} .
\end{aligned}
$$

Lemma 4.1. Suppose $\widetilde{G}$ is a covering of $G$. Let $\tilde{h}_{t}$ and $h_{t}$ denote the heat kernels of $\widetilde{G}$ and $G$, respectively. Then

$$
\sum_{x \in \pi^{-1}(u)} \sum_{y \in \pi^{-1}(v)} \tilde{h}_{t}(x, y)=\sqrt{\left|\pi^{-1}(u)\right|\left|\pi^{-1}(v)\right|} h_{t}(u, v) .
$$

Proof. A direct calculation shows that

$$
h_{t}(u, v)=e^{-t} \sum_{r} S_{r}(u, v) \frac{t^{r}}{r !}
$$

where $S_{r}$ is the sum of weights of all $r$-walks joining $u$ and $v$. We want to show that the total weights of the paths in $\widetilde{G}$ lifted from $p_{r}$ (i.e. whose image in $G$ is $p_{r}$ ) is exactly the weight of $p_{r}$ in $G$ multiplied by $\sqrt{\left|\pi^{-1}\left(u_{0}\right)\right|\left|\pi^{-1}\left(u_{r}\right)\right|}$. Let $p_{r-1}$ denote the walk $u_{0}, \ldots, u_{r-1}$. Suppose $u_{r-1} \neq u_{r}$. For each path $\tilde{p}_{r-1}$ lifted from $p_{r-1}$, its extensions to paths lifted from $p_{r}$ has total weights

$$
\begin{aligned}
& -w\left(\tilde{p}_{r-1}\right) \sum_{z \in \pi^{-1}\left(u_{r}\right)} \frac{\tilde{\phi}\left(\tilde{u}_{r-1}\right) \tilde{P}\left(\tilde{u}_{r-1}, z\right)+\tilde{\phi}(z) \tilde{P}\left(z, \tilde{u}_{r-1}\right)}{2 \sqrt{\tilde{\phi}\left(\tilde{u}_{r-1}\right) \tilde{\phi}(z)}} \\
& =-w\left(\tilde{p}_{r-1}\right) \sum_{z \in \pi^{-1}\left(u_{r}\right)} \frac{\frac{\phi\left(u_{r-1}\right)}{\left|\pi^{-1}\left(u_{r-1}\right)\right|} \tilde{P}\left(\tilde{u}_{r-1}, z\right)+\frac{\phi\left(u_{r}\right)}{\left|\pi^{-1}\left(u_{r}\right)\right|} \tilde{P}\left(z, \tilde{u}_{r-1}\right)}{2 \sqrt{\frac{\phi\left(u_{r-1}\right) \phi\left(u_{r}\right)}{\left|\pi^{-1}\left(u_{r-1}\right)\right|\left|\pi^{-1}\left(u_{r}\right)\right|}}} \\
& =\frac{-w\left(\tilde{p}_{r-1}\right) \sqrt{\left|\pi^{-1}\left(u_{r-1}\right)\right|\left|\pi^{-1}\left(u_{r}\right)\right|}}{2 \sqrt{\phi\left(u_{r-1}\right) \phi\left(u_{r}\right)}} \sum_{z \in \pi^{-1}\left(u_{r}\right)}\left(\frac{\phi\left(u_{r-1}\right)}{\left|\pi^{-1}\left(u_{r-1}\right)\right|} \tilde{P}\left(\tilde{u}_{r-1}, z\right)+\frac{\phi\left(u_{r}\right)}{\left|\pi^{-1}\left(u_{r}\right)\right|} \tilde{P}\left(z, \tilde{u}_{r-1}\right)\right)
\end{aligned}
$$

We will return later to the last expression and now consider the two sums contained therein: 
(i)

$$
\begin{aligned}
\sum_{z \in \pi^{-1}\left(u_{r}\right)} \frac{\phi\left(u_{r-1}\right)}{\left|\pi^{-1}\left(u_{r-1}\right)\right|} \tilde{P}\left(\tilde{u}_{r-1}, z\right) & =\frac{\phi\left(u_{r-1}\right)}{\left|\pi^{-1}\left(u_{r-1}\right)\right|} \sum_{z \in \pi^{-1}\left(u_{r}\right)} \frac{w\left(\tilde{u}_{r-1}, z\right)}{d_{\tilde{u}_{r-1}}} \\
& =\frac{\phi\left(u_{r-1}\right)}{d_{u_{r-1}}\left|\pi^{-1}\left(u_{r-1}\right)\right|} w\left(u_{r-1}, u_{r}\right) \\
& =\frac{\phi\left(u_{r-1}\right)}{\left|\pi^{-1}\left(u_{r-1}\right)\right|} P\left(u_{r-1}, u_{r}\right) .
\end{aligned}
$$

(ii)

$$
\begin{aligned}
\sum_{z \in \pi^{-1}\left(u_{r}\right)} \frac{\phi\left(u_{r}\right)}{\left|\pi^{-1}\left(u_{r}\right)\right|} \tilde{P}\left(z, \tilde{u}_{r-1}\right) & =\frac{\phi\left(u_{r}\right)}{\left|\pi^{-1}\left(u_{r}\right)\right|} \sum_{z \in \pi^{-1}\left(u_{r}\right)} \frac{w\left(z, \tilde{u}_{r-1}\right)}{d_{z}} \\
& =\frac{\phi\left(u_{r}\right)}{m d_{u_{r}}} \sum_{z \in \pi^{-1}\left(u_{r}\right)} w\left(z, \tilde{u}_{r-1}\right) \\
& =\frac{\phi\left(u_{r}\right)}{d_{u_{r}}\left|\pi^{-1}\left(u_{r-1}\right)\right|} w\left(u_{r}, u_{r-1}\right) \\
& =\frac{\phi\left(u_{r}\right)}{\left|\pi^{-1}\left(u_{r-1}\right)\right|} P\left(u_{r}, u_{r-1}\right) .
\end{aligned}
$$

Returning to the expression from before with the sums simplified we have:

$$
\begin{gathered}
\frac{-w\left(\tilde{p}_{r-1}\right) \sqrt{\mid \pi^{-1}\left(u_{r-1}\right)}|| \pi^{-1}\left(u_{r}\right) \mid}{2 \sqrt{\phi\left(u_{r-1}\right) \phi\left(u_{r}\right)}}\left(\frac{\phi\left(u_{r-1}\right)}{\left|\pi^{-1}\left(u_{r-1}\right)\right|} P\left(u_{r-1}, u_{r}\right)+\frac{\phi\left(u_{r}\right)}{\left|\pi^{-1}\left(u_{r-1}\right)\right|} P\left(u_{r}, u_{r-1}\right)\right) \\
=-w\left(\tilde{p}_{r-1}\right) \frac{\sqrt{\left|\pi^{-1}\left(u_{r}\right)\right|}}{\sqrt{\left|\pi^{-1}\left(u_{r-1}\right)\right|}}\left(\frac{\phi\left(u_{r-1}\right) P\left(u_{r-1}, u_{r}\right)+\phi\left(u_{r}\right) P\left(u_{r}, u_{r-1}\right)}{2 \sqrt{\phi\left(u_{r-1}\right) \phi\left(u_{r}\right)}}\right)
\end{gathered}
$$

By summing over all $\tilde{p}_{r-1}$, we have

$$
\sum_{x \in \pi^{-1}(u)} \sum_{y \in \pi^{-1}(v)} S_{r}(x, y)=\sqrt{\left|\pi^{-1}(u)\right|\left|\pi^{-1}(v)\right|} S_{r}(u, v) .
$$

As a consequence of Lemma 4.1, we have

Corollary 4.2. Suppose $\widetilde{G}$ is a strong regular covering of $G$. Let $\tilde{h}_{t}$ and $h_{t}$ denote the heat kernels of $\widetilde{G}$ and $G$ respectively. For $x \in \pi^{-1}(u)$, we have

$$
\sum_{y \in \pi^{-1}(v)} \tilde{h}_{t}(x, y)=\sqrt{\frac{\left|\pi^{-1}(v)\right|}{\left|\pi^{-1}(u)\right|}} h_{t}(u, v) .
$$


Corollary 4.3. Suppose $G$ is a distance regular graph which is a covering of a path $P$ with vertices $v_{0}, \ldots, v_{p}$ where $p=D(G)$, the diameter of $G$. Suppose $G$ and $P$ have heat kernels $\tilde{h}_{t}$ and $h_{t}$ respectively. For any two vertices $x$ and $y$ in $G$ with distance $d(x, y)=r$, we have

$$
\tilde{h}_{t}=\sqrt{\left|\pi^{-1}\left(v_{r}\right)\right|} h_{t}\left(v_{0}, v_{r}\right) .
$$

The following theorem is from [3]. The proof in the directed case follows exactly as in the undirected case and is offered here for the sake of completeness.

Theorem 4.4. Suppose $\widetilde{G}$ is a strong regular covering of $G$. Let $v$ denote the vertex of $G$ with preimage in $\widetilde{G}$ consisting of one vertex. Then any eigenvalue $\lambda$ of $\widetilde{G}$ has multiplicity

$$
n \sum_{i} \frac{f_{i}^{2}(v)}{\left\|f_{i}\right\|^{2}}
$$

where $n=|V(\widetilde{G})|$ and the $f_{i}$ 's span the eigenspace of $\lambda$ in $G$. If the eigenvalue $\lambda$ has multiplicity 1 in $G$ with eigenfunction $f$, then the multiplicity of $\lambda$ in $\widetilde{G}$ is

$$
\frac{n f^{2}(v)}{\|f\|^{2}}
$$

Proof. Suppose $\widetilde{G}$ has heat kernel $\tilde{h}_{t}$ and $G$ has heat kernel $h_{t}$. Since $\widetilde{G}$ is a strong regular covering of $G$, we have

$$
\operatorname{Tr}\left(\tilde{h}_{t}\right)=\sum_{x \in V(\widetilde{G})} \tilde{h}_{t}(x, x)=n h_{t}(v, v)=n \sum_{j} e^{-t \lambda_{j}} \frac{f_{j}^{2}(v)}{\left\|f_{j}\right\|^{2}} .
$$

Therefore, the multiplicity of $\lambda_{j}$ in $\widetilde{G}$ is exactly

$$
\frac{n f_{j}^{2}}{\left\|f_{j}\right\|^{2}}
$$

if the multiplicity of $\lambda$ in $G$ is 1 . In general, the multiplicity of $\lambda$ in $\widetilde{G}$ is

$$
n \sum_{i} \frac{f_{i}^{2}(v)}{\left\|f_{i}\right\|^{2}}
$$

where the $f_{i}$ 's span the eigenspace of $\lambda$ in $G$.

\subsection{The Heat Kernel of the $k$-regular Tree}

Let $T_{k}$ be an in Example 2.1 and $\mathcal{P}_{+}$be as in Example 3.1. Using the calculations in Example 3.1 and [3] we have that the heat kernel $h_{t}^{(l)}$ of $P^{(l)}$ satisfies

$$
h_{t}^{(l)}(0,0)=\sum_{j=1}^{l-1} \frac{e^{-t\left(1-\frac{2 \sqrt{\sigma-1}}{\sigma} \cos \frac{j \pi}{l}\right)} \sin ^{2} \frac{j \pi}{l}}{\frac{l \sigma}{2(\sigma-1)}\left(1-\frac{4(\sigma-1)}{\sigma^{2}} \cos ^{2} \frac{j \pi}{l}\right)}+\frac{1}{\left\|f_{0}\right\|^{2}}+\frac{1}{\left\|f_{l}\right\|^{2}} .
$$

THE ELECTRONiC JOURNAL OF COMBINATORICs 16 (2009), \#R31 
When $l$ approaches infinity, the heat kernel $h_{t}$ of $\mathcal{P}_{+}$satisfies:

$$
h_{t}(0,0)=\frac{2 \sigma(\sigma-1)}{\pi} \int_{0}^{\pi} \frac{e^{-t\left(1-\frac{2 \sqrt{\sigma-1}}{\sigma} \cos x\right)} \sin ^{2} x}{\sigma^{2}-4(\sigma-1) \cos ^{2} x} d x .
$$

In general, for $a \geq 1$, we have

$$
h_{t}(0, a)=\frac{2 \sqrt{\sigma(\sigma-1)}}{\pi} \int_{0}^{\pi} \frac{e^{-t\left(1-\frac{2 \sqrt{\sigma-1}}{\sigma} \cos x\right)} \sin x[(\sigma-1) \sin (a+1) x-\sin (a-1) x]}{\sigma^{2}-4(\sigma-1) \cos ^{2} x} d x .
$$

Let $H_{t}^{(l)}$ denote the heat kernel of the subtree of $T_{k}$ spanned by the $l$-ball centered at $z$. We write

$$
H_{t}^{(l)}=H_{1, t}^{(l)}+H_{2, t}^{(l)}
$$

where $H_{i, t}^{(l)}$ is the restriction to $V_{i}, i=1,2$. Remember that the eigenfunctions in $V_{1}$ are lifts of the eigenfunctions from $\mathcal{P}_{+}^{(l)}$. Also, since $V_{2}$ contains eigenfunctions that vanish at $z$, we have that

$$
H_{t}^{(l)}(z, v)=H_{1, t}^{(l)}(z, v) .
$$

As in [3], we have the following.

Theorem 4.5. With the above notation, $H_{t}\left(z, v_{i}^{(n)}\right)=H_{1, t}\left(z, v_{i}^{(n)}\right)$ and they are equal to

$$
\frac{2}{\pi(\sigma-1)^{n / 2-1}} \int_{0}^{\pi} \frac{e^{-t\left(1-\frac{2 \sqrt{\sigma-1}}{\sigma} \cos x\right)} \sin x[(\sigma-1) \sin (n+1) x-\sin (n-1) x]}{\sigma^{2}-4(\sigma-1) \cos ^{2} x} d x .
$$

For the term corresponding to $V_{2}$, let $v=\left(v_{\sigma_{i}^{(n)}}\right)$ be an eigenvector of $L^{(l)}$, the Laplacian on $T_{k}^{(l)}$, with $v_{z}=0$. Let $\lambda$ be its eigenvalue. Then the equation $v L^{(l)}=\lambda v$ implies (in what follows we take sums with $\left.\sigma_{j}^{(n-1)} \sim \sigma_{i}^{(n)}\right)$ :

$$
\begin{aligned}
\sum v_{\sigma_{i}^{(1)}} & =0 \\
v_{\sigma_{i}^{(1)}}-\frac{1}{\sqrt{\mu}(\rho+1)} \sum_{\sigma_{j}^{(2)} \sim \sigma_{i}^{(1)}} v_{\sigma_{j}^{(2)}} & =\lambda v_{\sigma_{i}^{(1)},} \\
-\frac{1}{\sqrt{\mu}(\rho+1)} v_{\sigma_{j}^{(n-1)}}+v_{\sigma_{i}^{(n)}}-\frac{1}{\sqrt{\mu}(\rho+1)} \sum_{\sigma_{j}^{(n+1)} \sim \sigma_{i}^{(n)}} v_{\sigma_{j}^{(n+1)}} & =\lambda v_{\sigma_{i}^{(n)}, n<l-1,} \\
-\frac{1}{\sqrt{\mu}(\rho+1)} v_{\sigma_{j}^{(l-2)}}+v_{\sigma_{i}^{(l-1)}}-\frac{1}{\sqrt{\mu(\rho+1)}} \sum_{\sigma_{j}^{(l)} \sim \sigma_{i}^{(l-1)}} v_{\sigma_{j}^{(l)}} & =\lambda v_{\sigma_{i}^{(l-1)}}, n=l-1, \\
-\frac{1}{\sqrt{\mu(\rho+1)}} v_{\sigma_{j}^{(l-1)}}+v_{\sigma_{i}^{(l)}} & =\lambda v_{\sigma_{i}^{(l)}}, n=l .
\end{aligned}
$$

The last equation implies that, if $\sigma_{i}^{(l)}$ and $\sigma_{j}^{(l)}$ are adjacent to the same vertex, then $v_{\sigma_{i}^{(l)}}=v_{\sigma_{j}^{(l)}}$. Also, each of the $k$-vertices $\sigma_{i}^{(n)}$ determines a subtree with root $z$. There are $k$ such subtrees. It is clear from the form of the equations that: 
1. Each subtree determines exactly the same system. Thus we need to find the eigenvalues of the square matrix of side $(k-1)^{l-1}-1, \Lambda_{l}$ with entries indexed by the vertices in the subtree:

$$
\Lambda_{l}\left(v_{\sigma_{i}^{(n)}}, v_{\sigma_{j}^{(m)}}\right)= \begin{cases}1, & \sigma_{i}^{(n)}=\sigma_{j}^{(m)} \\ \beta, & 1 \leq i, j \leq l-1, \sigma_{i}^{(n)} \sim \sigma_{j}^{(m)} \\ \gamma, & (i, j) \in\{(l, l-1),(l-1, l)\} \sigma_{i}^{(n)} \sim \sigma_{j}^{(m)} \\ 0, & \text { otherwise }\end{cases}
$$

where

$$
\beta=-\frac{1}{\sqrt{\mu}(\rho+1)}, \quad \gamma=-\frac{1}{\sqrt{\mu(\rho+1)}} .
$$

2. The connection between the solutions coming from dif and only iferent branches is given by the fact that

$$
\sum_{i \in S(n)} v_{\sigma_{i}^{(n)}}=0
$$

\section{The Heat Kernel For the one-dimensional weighted lattice graph}

We consider the homesick random walk with parameter $\lambda$ on the infinite path $\mathcal{P}$ (the one-dimensional lattice) as in [6]. More precisely, $V(\mathcal{P})=\mathbb{Z}$ with base vertex 0 . The weights are defined as follows:

$$
w(i, j)= \begin{cases}\frac{1}{2}, & i=0, j= \pm 1, \\ \frac{1}{\mu+1}, & i>0, j=i+1, \quad \text { or } i<0, j=i-1 \\ \frac{\mu}{\mu+1}, & i>0, j=i-1, \quad \text { or } i<0, j=i+1 \\ 0, & \text { otherwise }\end{cases}
$$

Let $\mathcal{P}_{+}$be the infinite ray with $V\left(\mathcal{P}_{+}\right)=\mathbb{N}$, base vertex 0 and the weights are defined as follows:

$$
w(i, j)=\left\{\begin{array}{cl}
1, & i=0, j=1 \\
\frac{2}{\mu+1}, & i>0, j=i+1 \\
\frac{2 \mu}{\mu+1}, & i>0 \\
0, & \text { otherwise. }
\end{array}\right.
$$

It is a direct calculation to show that the map

$$
\pi: V(\mathcal{P}) \rightarrow V\left(\mathcal{P}_{+}\right), \quad \pi(i)=|i|
$$


is a covering map.

For any $l>0$, let $\mathcal{P}^{(l)}$ be the subgraph of $\mathcal{P}$ with vertex set $V\left(\mathcal{P}^{(l)}\right)=\{0, \pm 1, \ldots, \pm l\}$. The weights are the same as in $\mathcal{P}$ except that

$$
w(-l,-l+1)=w(l, l-1)=1 .
$$

Also, we define the subgraph $\mathcal{P}_{+}^{(l)}$ of $\mathcal{P}_{+}$with the vertex set $V\left(\mathcal{P}_{+}^{(l)}\right)=\{0,1, \ldots, l\}$. Again, the weights are the same as in $\mathcal{P}_{+}$except that $w(l, l-1)=2$. Then the map $\pi$ restricts to a cover from $\mathcal{P}^{(l)}$ to $\mathcal{P}_{+}^{(l)}$. As in Example 3.1, the Laplacian of $\mathcal{P}_{+}^{(l)}$ is given by:

$$
\mathcal{L}^{(l)}(i, j)=\left\{\begin{aligned}
1 ; & i=j, \\
-\sqrt{\frac{1}{k}} ; & (i, j)=(0,1) \text { or }(1,0), \\
-\frac{\sqrt{k-1}}{k} ; & |i-j|, 0<i, j<l, \\
-\sqrt{\frac{k-1}{k} ;} & (i, j)=(l-1, l) \text { or }(l, l-1), \\
0 ; & \text { otherwise. }
\end{aligned}\right.
$$

where $k=\frac{\mu+1}{\mu}$. As in Example 3.1, the eigenvalues of $\mathcal{L}^{(l)}$ are 0,2 and

$$
\lambda_{n}=1-\frac{2 \sqrt{k-1}}{k} \cos \frac{\pi n}{l}=1-\frac{2 \sqrt{\mu}}{\mu+1} \cos \frac{\pi n}{l}, \text { for } n=1, \ldots, l-1 .
$$

The eigenfunction $\phi_{0}$ associated with the eigenvalue 0 can be written as $f_{0} /\left\|f_{0}\right\|$ where

$$
\begin{aligned}
& f_{0}(0)=1 \\
& f_{0}(p)=\sqrt{\frac{\mu+1}{\mu^{p}}}, \text { for } 1 \leq p \leq l-1, \\
& f_{0}(l)=\frac{1}{\sqrt{\mu^{l-1}}} .
\end{aligned}
$$

We now consider the eigenfunction $\phi_{l}$ which corresponds to the eigenvalue 2 . In this case, $\phi_{l}=f_{l} /\left\|f_{l}\right\|$, where $f_{l}$ is defined as follows:

$$
\begin{aligned}
& f_{l}(0)=1 \\
& f_{l}(p)=(-1)^{p} \sqrt{\frac{\mu+1}{\mu^{p}}}, \text { for } 1 \leq p \leq l-1, \\
& f_{l}(l)=(-1)^{l} \frac{1}{\sqrt{\mu^{l-1}}} .
\end{aligned}
$$


For each $n \in\{1,2, \ldots, l-1\}$ there is an eigenfunction $\phi_{n}$ associated with the eigenvalue $\lambda_{n}$. We can write $\phi_{n}=f_{n} /\left\|f_{n}\right\| \in L^{2}\left(\mathcal{P}_{+}^{(l)}\right.$ where

$$
\begin{aligned}
& f_{n}(0)=\sqrt{\mu+1} \sin \frac{\pi n}{l}, \\
& f_{n}(p)=\sin \frac{\pi n(p+1)}{l}-\mu \sin \frac{\pi n(p-1)}{l}, \text { for } 1 \leq p \leq l-1, \\
& f_{n}(l)=\sqrt{\mu(\mu+1)} \sin \frac{\pi n}{l} .
\end{aligned}
$$

For $n=1, \ldots, l-1$,

$$
\left\|f_{j}\right\|^{2}=l(\mu+1)^{2}\left(1-\frac{4 \mu}{(\mu+1)^{2}} \cos ^{2} \frac{n \pi}{l}\right) .
$$

Therefore the heat kernel $h_{t}^{(l)}$ of $P_{+}^{(l)}$ satisfies

$$
h_{t}^{(l)}(0,0)=\sum_{j=1}^{l-1} \frac{e^{-t\left(1-\frac{2 \sqrt{\mu}}{\mu+1} \cos \frac{j \pi}{l}\right)} \sin ^{2} \frac{j \pi}{l}}{\frac{l(\mu+1)}{2}\left(1-\frac{4 \mu}{(\mu+1)^{2}} \cos ^{2} \frac{j \pi}{l}\right)}+\frac{1}{\left\|f_{0}\right\|^{2}}+\frac{1}{\left\|f_{l}\right\|^{2}} .
$$

Let $H_{t}^{(l)}$ denote the heat kernel of $\mathcal{P}^{(l)}$. Then, as in Example 4.1, $H_{t}^{(l)}=H_{1, t}^{(l)}+H_{1, t}^{(l)}$. Since $H_{1, t}^{(l)}(0, a)$ depends only on $|a|$, we have that

Proposition 5.1. For each $a \in \mathbb{Z}$, the heat kernel $H_{t}$ of the homesick random walk on the infinite path satisfies

$$
H_{t}(0, a)=\frac{\mu \sqrt{2(\mu+1)}}{\pi} \int_{0}^{\pi} \frac{e^{-t\left(1-\frac{2 \sqrt{\mu}}{\mu+1} \cos x\right)} \sin x\left[\frac{1}{\mu} \sin (|a|+1) x-\sin (|a|-1) x\right]}{\mu^{2}+2 \mu+1-4 \mu \cos ^{2} x} d x .
$$

Furthermore, in this case $H_{t}(0, a)=H_{1, t}(0, a)$.

Let $\lambda$ be an eigenvalue of $L^{(l)}$, the Laplacian on $\mathcal{P}^{(l)}$, whose eigenfuctions $\phi$ vanish at 0. Direct calculations show that:

1. $\phi(-a)=-\phi(a)$.

2. $\lambda$ is an eigenvalue of the matrix

$$
\Lambda^{(l)}(i, j)=\left\{\begin{aligned}
1, & \text { if } i=j, \\
-\frac{1}{\sqrt{\mu+1},} & \text { if } i, j \in\{-l,-(l-1)\}, i \neq j, \\
-\frac{\sqrt{\mu}}{\mu+1}, & \text { if }|i-j|=1, \text { and not in the last case, } \\
0, & \text { otherwise }
\end{aligned}\right.
$$

The substitution $r=\mu+1$, gives the matrix in Section 5 in [3], except the difference at the $2 \times 2$-block at the right low corner. 
Now the heat kernel of $\mathcal{P}^{(l)}$ has two summands:

$$
H_{t}^{(l)}(a, b)=H_{1, t}^{(l)}(a, b)+H_{2, t}^{(l)}(a, b),
$$

where the first summand involves the eigenvalues of the Laplacian with eigenfunction not vanishing at the vertex 0 and the second the eigenvalues with the corresponding eigenfunction being zero at 0 . There are $l$ eigenvalues in the second summand, so there are $2 l+1$ eigenvalues in the first summand. Since all the eigenvalues in the first summand have multiplicity 1 and their eigenfunctions are their projections to the corresponding eigenfunctions to $\mathcal{P}_{+}^{(l)}$,

$$
H_{1, t}^{(l)}(a, b)=h_{t}^{(l)}(|a|,|b|) .
$$

As in Section 5 in [3], taking $l \rightarrow \infty$ :

$$
\begin{aligned}
& H_{1, t}^{(l)}(a, b)=h_{t}^{(l)}(|a|,|b|)= \\
& \frac{2 \mu^{2}}{\pi} \int_{0}^{\pi} \frac{e^{-t\left(1-\frac{2 \sqrt{\mu}}{\mu+1} \cos x\right)}[\sin ((|a|+1) x)-\sin (|a|-1) x][\sin ((|b|+1) x)-\sin (|b|-1) x]}{\mu^{2}+2 \mu+1-4 \mu \cos ^{2} x} d x .
\end{aligned}
$$

For the calculations of $H_{2, t}(a, b)$, we use the calculations of Section 5 in [3], because as $l \rightarrow \infty$, the limit of $\Lambda^{(l)}$ and the Laplacian of the path which is covered by the $(\mu+1)$ regular tree in [3] are the same (even though $\mu$ might not be an positive integer). Thus

$$
\begin{aligned}
& H_{2, t}^{(l)}(a, b)= \\
& \frac{2}{\pi} \int_{0}^{\pi} \frac{e^{-t\left(1-\frac{2 \sqrt{\mu}}{\mu+1} \cos x\right)}[\sin ((|a|+1) x)-\sin (|a|-1) x][\sin ((|b|+1) x)-\sin (|b|-1) x]}{\mu^{2}+2 \mu+1-4 \mu \cos ^{2} x} d x
\end{aligned}
$$

and the sign is positive if $a$ and $b$ have the same sign and negative otherwise.

Summarizing, we have the following

Theorem 5.2. For the homesick random walk with parameter $\mu$ on the infinite line, the heat kernel $H_{t}(a, b)$ satisfies:

1. If $b=0$, then $H_{t}(a, 0)$ is

$$
\frac{\mu \sqrt{2(\mu+1)}}{\pi} \int_{0}^{\pi} \frac{e^{-t\left(1-\frac{2 \sqrt{\mu}}{\mu+1} \cos x\right)} \sin x\left[\frac{1}{\mu} \sin (a+1) x-\sin (a-1) x\right]}{\mu^{2}+2 \mu+1-4 \mu \cos ^{2} x} d x .
$$

2. If both $a$ and $b$ are not 0 , then $H_{t}(a, b)$ is:

$$
K \int_{0}^{\pi} \frac{e^{-t\left(1-\frac{2 \sqrt{\mu}}{\mu+1} \cos x\right)}[\sin ((|a|+1) x)-\sin (|a|-1) x][\sin ((|b|+1) x)-\sin (|b|-1) x]}{\mu^{2}+2 \mu+1-4 \mu \cos ^{2} x} d x
$$

where $K=\frac{2}{\pi}\left(\mu^{2}+\varepsilon\right)$ and $\varepsilon=\operatorname{sign}(a) \cdot \operatorname{sign}(b)$. 


\section{References}

[1] F. R. K. Chung, Spectral graph theory, CBMS Regional Conference Series in Mathematics, 92. Published for the Conference Board of the Mathematical Sciences, Washington, DC; by the American Mathematical Society, Providence, RI, 1997.

[2] F. R. K. Chung, Laplacians and the Cheeger inequality for directed graphs, Ann. of Comb., 9 (2005), 1-19.

[3] F. R. K. Chung and S.-T. Yau, Coverings, heat kernels, and spanning trees, Electron. J. Combin., 6 (1999), Research Paper 12.

[4] P. de la Harpe, Topics in geometric group theory, Chicago Lectures in Mathematics. University of Chicago Press, Chicago, IL, 2000.

[5] H. Kesten, Symmetric random walks on groups, Trans. Amer. Math. Soc. 92 (1959), $336-354$.

[6] R. Lyons, Random walks and the growth of groups, C. R. Acad. Sci. Paris Sér. I Math., 320 (1995), 1361-1366.

[7] H. Minc, Nonnegative matrices, Wiley-Interscience Publications, New York, 1988. 